finding is in agreement with other studies that have reported in vivo $\mathrm{O} 2$ content of the microvasculature. bEnd.3 cells cultured under $5 \mathrm{kPa}$ also exhibited lower intracellular glutathione content compared to cells cultured under $18 \mathrm{kPa}$ O2 ( 20.6 vs $29.9 \mathrm{mmol} / \mathrm{mg}$ protein, $\mathrm{n}=4, \mathrm{p}=0.003$ ). Furthermore, bEnd. 3 cells cultured under $5 \mathrm{kPa} \mathrm{O} 2$ exhibited greater sensitivity to OGD-induced cell death, with increased ratio of dead to live cells seen under 2 hour OGD $(0.42$ vs $0.05, n=3$, $\mathrm{p}=0.0002$ ).

Conclusions The majority of cell culture studies have conducted experiments under hyperoxia. The present study highlights the importance of conducting cell culture studies under physiological O2 levels, as demonstrated by the different phenotype resulted from long-term culture under physiological $\mathrm{O} 2$ levels.

This work was supported by Grant (FS/13/66/30445) from the British Heart Foundation. The authors declare no conflicts of interest.

\section{AN ASSESSMENT OF THE SMOKING CESSATION POLICY IN AN NHS TRUST}

${ }^{1}$ Sachin Ananth, ${ }^{1}$ Felicite Mukeshimana, ${ }^{1}$ Sanjida Rahman, ${ }^{2}$ Nicola Whiteley, ${ }^{2}$ Louisa Pierce, ${ }^{2}$ Raymond Anakwe. 'Imperial College School of Medicine; ${ }^{2}$ Imperial College Healthcare NHS Trust

\subsection{6/postgradmedj-2018-fpm.29}

Introduction Smoking is the second biggest risk factor for premature death in the UK, and it is a significant risk factor for many chronic illnesses. Approximately $25 \%$ of secondary care patients smoke, and so treating tobacco dependence would create an opportunity to improve the health of these patients. Referral to stop smoking services is the optimum way to induce smoking cessation- smokers who are referred to these services are four times more likely to quit. NICE guidelines set out the standard of smoking cessation support in secondary care. This audit aimed to assess the compliance of an NHS Trust's smoking cessation policy with these NICE guidelines.

Subjects and methods The NICE baseline assessment tool was used to assess the compliance of the Trust's smoking cessation policy with the NICE guidelines. To evaluate the implementation of the Trust's policy in clinical practice, snapshot audits of the medical notes of current inpatients and outpatients were undertaken. Medical notes were collected from at least 2 medical specialties and 2 surgical specialties. The notes were assessed for documented smoking status in all patients, and offer of nicotine-replacement therapy (NRT) and referral to a stop smoking service in current smokers.

Results The Trust's smoking cessation policy was partially compliant with the NICE guidelines; significant gaps in the Trust's policy included smoking cessation in outpatients and the training of all staff to deliver stop smoking advice. 14/27 (51.9\%) of outpatients and 54/80 (67.5\%) of inpatients had smoking status documented in their notes. Documentation varied widely across the different specialties, ranging from $0 \%$ of Neurology and ENT outpatients, to $100 \%$ of Respiratory inpatients. $7 / 19(36.8 \%)$ of current smokers had a documented offer of NRT, while 4/19 (21.1\%) of current smokers had a documented offer of referral to a stop smoking service. Of the 3 current smokers in the Respiratory ward, none were offered NRT and 1 was offered a referral to a stop smoking service.

Conclusions While the Trust's smoking cessation policy partially complied with the NICE guidelines, implementation of the Trust policy was insufficient. There were low rates of smoking status documentation in many specialties, and so healthcare staff should be encouraged to ask about smoking status, even if it may not seem relevant to the patient's presenting complaint. Few smokers were offered referral to stop smoking services. Greater awareness of these services is needed, so that smokers receive appropriate smoking cessation support, ensuring that opportunities to improve the health of the nation are not missed.

\section{THE BENEFITS OF AN INTEGRATED EYE CLINIC LIAISON OFFICER IN IMPROVING PATIENT OUTCOMES IN OPHTHALMOLOGY}

Jennifer Bacon, Hayley Coates, Nitin Gupta. West Suffolk NHS Foundation Trust, UK

\subsection{6/postgradmedj-2018-fpm.30}

Introduction The loss of sight can have a profound impact on a patient's independence and well-being. Giving patients the devastating news that they are losing their sight is very challenging and when receiving information, a patient often requires considerable support and time. Eye Clinic Liaison officers (ECLOs) are essential in providing patients, their families, and carers with practical and emotional support to help understand their diagnosis. They can help patients not only adapt to living with sight loss, but also to ensure they do not become isolated and retain their independence. ECLOs provide a vital role in bridging the gap between health and social care services, such as fall prevention, job retention, education and social services, enabling early intervention. The service demonstrates how health and social care can work together effectively and this supports the NHS outcomes framework. At the West Suffolk NHS Foundation Trust we have an integrated ECLO. This has proven to reduce DNA's, reduce clinician time per patient, help patient compliance with treatment, and streamline the process for sight registrations. The Royal college of Ophthalmologists recognised the importance of the role of ECLOs in 2007, in its guidelines to commissioners and clinicians, which recommended the use of ECLO's as part of Age-Related macular degeneration services.

Methods In order to investigate the aims and objectives, evidence was gathered from Ophthalmologists, Patients and other eye clinic staff, with satisfaction surveys filled by those whom accessed or referred patients to the ECLO service.

Results Surveys were collated and feedback was positive. Having an integrated ECLO has meant timely referrals to appropriate services, providing access to support, and also services such as low visual aids and mental health. Direct access to mental health services reduces the need for GP appointments, meaning that those who require help receive it sooner. Early falls prevention and referrals into the community team have reduced the number of falls for patients with visual impairment, reducing hospital admissions. 95\% of surveys received from staff, working with ECLOs, said that it improved the patients experience and reduced time spent in 